\title{
On More General Lipschitz Spaces
}

\author{
Dorothee D. Haroske
}

Abstract. The present paper deals with (logarithmic) Lipschitz spaces of type $\operatorname{Lip}_{p, q}^{(1,-\alpha)}(1 \leq$ $p \leq \infty, 0<q \leq \infty, \alpha>\frac{1}{q}$ ). We study their properties and derive some (sharp) embedding results. In that sense this paper can be regarded as some continuation and extension of our papers $[8,9]$, but there are also connections with some recent work of Triebel concerning Hardy inequalities and sharp embeddings. Recall that the nowadays almost 'classical' forerunner of investigations of this type is the Brézis-Wainger result [6] about the 'almost' Lipschitz continuity of elements of the Sobolev spaces $H_{p}^{1+\frac{n}{p}}\left(\mathbb{R}^{n}\right)$ when $1<p<\infty$.

Keywords: Limiting embeddings, Lipschitz spaces, function spaces

AMS subject classification: 26 A 16, 46 E 35, 26 A 15

\section{Introduction}

The present paper arose in connection with our recent papers $[8,9]$ as well as after some discussion with $\mathrm{H}$. Triebel about this subject. Concerning our joint papers [8, 9] with D. E. Edmunds, we were mainly led by two different questions to study certain spaces of Lipschitz type and related embeddings.

On the one hand, compact embeddings of the type

$$
i d: B_{p_{1}, q_{1}}^{s_{1}}(\Omega) \longrightarrow B_{p_{2}, q_{2}}^{s_{2}}(\Omega)
$$

have been investigated for a long time already. Here $\Omega \subset \mathbb{R}^{n}$ is a bounded $C^{\infty}$ domain, and $B_{p_{i}, q_{i}}^{s_{i}}$ are the usual Besov spaces with $0<p_{i}, q_{i} \leq \infty$ and $s_{i} \in \mathbb{R} \quad(i=1,2)$. Embedding (1) is compact if

$$
0<p_{1}, p_{2} \leq \infty, \quad s_{1}-s_{2}>n \max \left(\frac{1}{p_{1}}-\frac{1}{p_{2}}, 0\right), \quad 0<q_{1}, q_{2} \leq \infty .
$$

The question now arises what happens when (2) is replaced by

$$
s_{1}-\frac{n}{p_{1}}=s_{2}-\frac{n}{p_{2}}, \quad 0<p_{1} \leq p_{2} \leq \infty, \quad 0<q_{1}, q_{2} \leq \infty
$$

the so-called limiting case. Clearly, embedding (1) is no longer compact. However, modifying the setting slightly, say, enlarging the target space sufficiently carefully (where the initial space is assumed to be fixed now), may lead to compact limiting embeddings.

Dorothee D. Haroske: Friedrich Schiller Univ., Math. Inst., D-07740 Jena

haroske@minet.uni-jena.de 
One approach was given by Edmunds and Triebel in [10: Section 3.4], changing the integrability $p_{2}$ of the target space slightly, where now spaces similar to the well-known Lorentz-Zygmund spaces $L_{p}(\log L)_{a}(\Omega) \quad(a \in \mathbb{R})$ appear. A parallel result, concerning the (weighted) $\mathbb{R}^{n}$-setting can be found in $\left[\frac{1}{2}, \frac{1}{3}\right]$.

Another idea to recover compactness of (1) when $s_{1}-\frac{n}{p_{1}}=s_{2}-\frac{n}{p_{2}}$, is to decrease the smoothness of the target space in such a way that the embedding becomes compact again, but the smoothness $s_{2}$ is preserved; i.e. we stick at the limiting situation. In that way one quite naturally arrives at the introduction of new spaces with additional 'logarithmic smoothness'. As an example one may consider the case $s_{2}=1$ and $p_{2}=\infty$. It turns out that in the case of $B$-spaces there is an interplay between the (usually neglected) $q$-parameters and the additional logarithmic smoothness. This result is somewhat surprising in our opinion, though similar results were obtained before (cf. [11]).

The second reason to deal with spaces of 'logarithmic smoothness' in more detail, is the well-known and celebrated result of Brézis and Wainger [6] in which it was shown that every function $u$ in $H_{p}^{1+\frac{n}{p}}\left(\mathbb{R}^{n}\right)$ is 'almost' Lipschitz-continuous, in the sense that, for all $x, y \in \mathbb{R}^{n}$ with $0<|x-y|<\frac{1}{2}$,

$$
|u(x)-u(y)| \leq c|x-y||\log | x-\left.y\right|^{\frac{1}{p^{\prime}}}\left\|u \mid H_{p}^{1+\frac{n}{p}}\left(\mathbb{R}^{n}\right)\right\| .
$$

Here $c$ is a constant independent of $x, y$ and $u$, and $\frac{1}{p^{\prime}}+\frac{1}{p}=1$. Our aim in [9] was to investigate how 'sharp' this result is (concerning the exponent of the log-term), as well as to look for possible extensions to the wider scale of $F$-spaces and parallel results for $B$-spaces. We found that the exponent $\frac{1}{p^{\prime}}$ is sharp in the $F$-setting, whereas in case of $B$-spaces the sharp exponent turned out to be $\frac{1}{q^{\prime}}$. As already mentioned above, this important role played by the $q$-parameter is rather unusual.

Moreover, (3) also suggests some definition of 'logarithmic' Lipschitz spaces in the following way: some $f \in C\left(\mathbb{R}^{n}\right)$ belongs to $\operatorname{Lip}^{(1,-\alpha)}\left(\mathbb{R}^{n}\right) \quad(\alpha \geq 0)$ if

$$
\left\|f\left|\operatorname{Lip}^{(1,-\alpha)}\left(\mathbb{R}^{n}\right)\|:=\| f\right| L_{\infty}\left(\mathbb{R}^{n}\right)\right\|+\sup _{0<|h|<\frac{1}{2}} \sup _{x \in \mathbb{R}^{n}} \frac{\left|\left(\Delta_{h} f\right)(x)\right|}{|h||\log | h||^{\alpha}}
$$

is finite. Here $\Delta_{h}$ is given by $\left(\Delta_{h} f\right)(x)=f(x+h)-f(x) \quad\left(x, h \in \mathbb{R}^{n}\right)$, as usual. Thus the Brézis-Wainger result (3) (together with the sharpness assertion [9: Theorem 2.1]) reads as $H_{p}^{1+\frac{n}{p}}\left(\mathbb{R}^{n}\right) \hookrightarrow \operatorname{Lip}^{(1,-\alpha)}\left(\mathbb{R}^{n}\right)$ if, and only if, $\alpha \geq \frac{1}{p^{\prime}}$, where $1<p<\infty$ and $\frac{1}{p}+\frac{1}{p^{\prime}}=1$. Studying (logarithmic) Lipschitz spaces in greater detail, one observes that - simply by construction - (4) can be understood as definition for spaces Lip ${ }^{(1,-\alpha)}=$ $\operatorname{Lip}_{\infty, \infty}^{(1,-\alpha)}(\alpha \geq 0)$, whereas the more general setting leads to spaces $\operatorname{Lip}_{p, q}^{(1,-\alpha)}(1 \leq p \leq$ $\left.\infty, 0<q \leq \infty, \alpha>\frac{1}{q}\right)$ given by

$$
\left\|f\left|\operatorname{Lip}_{p, q}^{(1,-\alpha)}\left(\mathbb{R}^{n}\right)\|=\| f\right| L_{p}\left(\mathbb{R}^{n}\right)\right\|+\left\{\begin{array}{ll}
\left(\int_{0}^{1 / 2}\left[\frac{\omega(f, t)_{p}}{t|\log t|^{\alpha}}\right]^{q} \frac{d t}{t}\right)^{\frac{1}{q}} & \text { if } q<\infty \\
\sup _{0<t<\frac{1}{2}} \frac{\omega(f, t)_{p}}{t|\log t|^{\alpha}} & \text { if } q=\infty
\end{array}\right\}<\infty
$$


where $\omega(f, t)_{p}=\sup _{|h| \leq t}\left\|\Delta_{h} f \mid L_{p}\left(\mathbb{R}^{n}\right)\right\|$ for $t>0$ and $f \in L_{p}\left(\mathbb{R}^{n}\right)$. Note that in this notation (4) can be rewritten as

$$
\left\|f\left|\operatorname{Lip}_{\infty, \infty}^{(1,-\alpha)}\left(\mathbb{R}^{n}\right)\|\sim\| f\right| L_{\infty}\left(\mathbb{R}^{n}\right)\right\|+\sup _{0<t<\frac{1}{2}} \frac{\omega(f, t)_{\infty}}{t|\log t|^{\alpha}}
$$

One may ask now which embedding results can be derived for such spaces when $p<$ $\infty, q<\infty$, and compare the outcome with the case already studied, i.e. for $p=q=\infty$. We will follow this question in the present paper.

It seems that there are also connections with Hardy inequalities and sharp embeddings as some related investigations by Triebel suggest.

Finally, let us briefly mention that these logarithmic Lipschitz spaces appear in many more connections, e.g. when studying (generalised) moduli of smoothness and related inequalities (see [3, 7]). Furthermore, these spaces seem involved when characterising the regularity of solutions in stationary problems (see [17]) and when investigating hydrodynamics in Besov spaces (cf. [20]). Thus it is not only of inner-mathematical interest to study such spaces in greater detail, but also in view of applications. They are, however, out of the scope of the present paper.

At first, in Section 1, we will briefly recall some fundamentals about the function spaces in question. Next, in Section 2, we derive some results about equivalent norms for the spaces under consideration. In Section 3 we investigate related 'sharp' embeddings between different spaces. Finally, in Section 4, we will briefly compare our approach with a few others, dealing with spaces of logarithmic smoothness, too.

Acknowledgements. It is a pleasure for me to give my thanks to Prof. Dr. H. Triebel who gave me opportunity to discuss the problem with him several times.

\section{Preliminaries}

We start with recalling some definitions. All further details may be found in [8, 9].

Let $\mathbb{R}^{n}$ be Euclidean $n$-space. Given two (quasi-) Banach spaces $X$ and $Y$, we write $X \hookrightarrow Y$ if $X \subset Y$ and the natural embedding of $X$ into $Y$ is continuous. All unimportant positive constants will be denoted by $c$, occasionally with subscripts. For some $a \in \mathbb{R}$ put $a_{+}:=\max (a, 0)$. Moreover, for $0<r \leq \infty$ the number $r^{\prime}$ is given by $\frac{1}{r^{\prime}}:=\left(1-\frac{1}{r}\right)_{+}$.

Let $C\left(\mathbb{R}^{n}\right)$ be the space of all complex-valued bounded continuous functions on $\mathbb{R}^{n}$, equipped with the sup-norm as usual. If $m \in \mathbb{N}$, we define

$$
C^{m}\left(\mathbb{R}^{n}\right)=\left\{f: D^{\alpha} f \in C\left(\mathbb{R}^{n}\right) \text { for all }|\alpha| \leq m\right\}
$$

Here $D^{\alpha}$ are classical derivatives and $C^{m}\left(\mathbb{R}^{n}\right)$ is endowed with the norm

$$
\left\|f\left|C^{m}\left(\mathbb{R}^{n}\right)\left\|=\sum_{|\alpha| \leq m}\right\| D^{\alpha} f\right| L_{\infty}\left(\mathbb{R}^{n}\right)\right\|
$$


Recall the concept of the difference operator $\Delta_{h}^{m}\left(m \in \mathbb{N}_{0}, h \in \mathbb{R}^{n}\right)$ : Let $f$ be an arbitrary function on $\mathbb{R}^{n}$. Then

$$
\left.\begin{array}{rl}
\left(\Delta_{h}^{1} f\right)(x) & =f(x+h)-f(x) \\
& \vdots \\
\left(\Delta_{h}^{m+1} f\right)(x) & =\Delta_{h}^{1}\left(\Delta_{h}^{m} f\right)(x)
\end{array}\right\}
$$

where $x, h \in \mathbb{R}^{n}$. Moreover, for some $r \in \mathbb{N}$ and $1 \leq p \leq \infty$, the $r$-th modulus of smoothness of a function $f \in L_{p}\left(\mathbb{R}^{n}\right)$ is defined by

$$
\omega_{r}(f, t)_{p}=\sup _{|h| \leq t}\left\|\Delta_{h}^{r} f \mid L_{p}\left(R^{n}\right)\right\| \quad(t>0)
$$

(see [3: Chapter 5/Definition 4.2, p. 332] or [7: Chapter 2/§7, pp. 44-46]). Sometimes, when there is no danger of confusion, we may write $\omega(f, t)_{p}$ instead of $\omega_{1}(f, t)_{p}$ and $\omega_{r}(f, t)$ instead of $\omega_{r}(f, t)_{\infty}$.

Definition 1. Let $1 \leq p \leq \infty, 0<q \leq \infty$ and $\alpha>\frac{1}{q}$. Then $\operatorname{Lip}_{p, q}^{(1,-\alpha)}\left(\mathbb{R}^{n}\right)$ is defined as the set of all $f \in L_{p}\left(\mathbb{R}^{n}\right)$ such that

$$
\left\|f\left|\operatorname{Lip}_{p, q}^{(1,-\alpha)}\left(\mathbb{R}^{n}\right)\|:=\| f\right| L_{p}\left(\mathbb{R}^{n}\right)\right\|+\left(\int_{0}^{1 / 2}\left[\frac{\omega(f, t)_{p}}{t|\log t|^{\alpha}}\right]^{q} \frac{d t}{t}\right)^{\frac{1}{q}}
$$

(with the usual modification if $q=\infty$ ) is finite.

Remark 2. Note that Definition 1 coincides with [8: Definition 4.1] when $q=\infty$, i.e. in our former notation we have $\operatorname{Lip}_{p}^{(1,-\alpha)}=\operatorname{Lip}_{p, \infty}^{(1,-\alpha)}(\alpha \geq 0,1 \leq p \leq \infty)$. Moreover, in case of $p=q=\infty, \alpha \geq 0$ we regain the logarithmic Lipschitz spaces $\operatorname{Lip}^{(1,-\alpha)}=\operatorname{Lip}_{\infty, \infty}^{(1,-\alpha)}$ introduced in [9], which for $\alpha=0$ collapse to the classical Lipschitz spaces.

The restriction $\alpha>\frac{1}{q}$ is quite natural as otherwise we have $\operatorname{Lip}_{p, q}^{(1,-\alpha)}=\{0\}$ only (see Remark 18 below). However, when $q=\infty$ we may also admit $\alpha=0$.

Remark 3. The spaces $\operatorname{Lip}_{\infty, \infty}^{(1,-\alpha)}\left(\mathbb{R}^{n}\right) \quad(\alpha \geq 0)$ can also be obtained as a special case of the more general spaces $C^{0, \sigma(t)}(\bar{\Omega})\left(\Omega \subseteq \mathbb{R}^{n}\right)$, which were introduced by Kufner, John and Fučík (see [15: Definition 7.2.12, p. 361]). Put $\sigma_{\alpha}(t)=t|\log t|^{\alpha}$, for small $t>0, \alpha \geq 0$, and $\sigma_{\alpha}(0):=0$. Then one easily checks that $\operatorname{Lip}_{\infty, \infty}^{(1,-\alpha)}\left(\mathbb{R}^{n}\right)=C^{0, \sigma_{\alpha}(t)}\left(\overline{\mathbb{R}^{n}}\right)$ (see [15: Definition 7.2.12, p. 361] for details).

Moreover, spaces of type $\operatorname{Lip}_{p, \infty}^{(1,-\alpha)}(\alpha=0)$ were introduced as $\operatorname{Lip}\left(1, L_{p}\right)$ by DeVore and Lorentz in [7: Chapter $2 / \S 9$, p. 51], where $\mathbb{R}^{n}$ is being replaced by some interval $[a, b] \subset \mathbb{R}$ and $0<p \leq \infty$.

We recall briefly the basic ingredients needed to introduce spaces of type $B_{p, q}^{s}$ and $F_{p, q}^{s}$. Leopold studied in [16] spaces of type $B_{p, q}^{(s, b)}(b \in \mathbb{R})$ which extend the scale of usual $B$-spaces in terms of smoothness. In order to compare related results later we give here the more general definition of $B_{p, q}^{(s, b)}$ instead of $B_{p, q}^{s}$. The Schwartz space 
$S\left(\mathbb{R}^{n}\right)$ and its dual $S^{\prime}\left(\mathbb{R}^{n}\right)$ of all complex-valued tempered distributions have their usual meaning here. Furthermore, $L_{p}\left(\mathbb{R}^{n}\right)$ with $0<p \leq \infty$ is the usual quasi-Banach space with respect to Lebesgue measure. Let $\varphi \in S\left(\mathbb{R}^{n}\right)$ be such that

$$
\operatorname{supp} \varphi \subset\left\{y \in \mathbb{R}^{n}:|y|<2\right\} \quad \text { and } \quad \varphi(x)=1 \text { if }|x| \leq 1 \text {, }
$$

put $\varphi_{0}=\varphi$ and for each $j \in \mathbb{N}$ let

$$
\varphi_{j}(x)=\varphi\left(2^{-j} x\right)-\varphi\left(2^{-j+1} x\right) .
$$

Then since $1=\sum_{j=0}^{\infty} \varphi_{j}(x)$ for all $x \in \mathbb{R}^{n}$, the $\left\{\varphi_{j}\right\}_{j=0}^{\infty}$ form a a dyadic partition of unity. Given any $f \in S^{\prime}\left(\mathbb{R}^{n}\right)$, we denote by $\mathcal{F} f$ and $\mathcal{F}^{-1} f$ its Fourier transform and its inverse Fourier transform, respectively.

Definition 4. Let $s \in \mathbb{R}, 0<q \leq \infty$, and let $\left\{\varphi_{j}\right\}$ be the above dyadic resolution of unity.

(i) Let $0<p \leq \infty$ and $b \in \mathbb{R}$. The space $B_{p, q}^{(s, b)}\left(\mathbb{R}^{n}\right)$ is the collection of all $f \in S^{\prime}\left(\mathbb{R}^{n}\right)$ such that

$$
\left\|f \mid B_{p, q}^{(s, b)}\left(\mathbb{R}^{n}\right)\right\|=\left(\sum_{j=0}^{\infty} 2^{j s q}(1+j)^{b q}\left\|\mathcal{F}^{-1} \varphi_{j} \mathcal{F} f \mid L_{p}\left(\mathbb{R}^{n}\right)\right\|^{q}\right)^{\frac{1}{q}}
$$

(with the usual modification if $q=\infty$ ) is finite.

(ii) Let $0<p<\infty$. The space $F_{p, q}^{s}\left(\mathbb{R}^{n}\right)$ is the collection of all $f \in S^{\prime}\left(\mathbb{R}^{n}\right)$ such that

$$
\left\|f\left|F_{p, q}^{s}\left(\mathbb{R}^{n}\right)\|=\|\left(\sum_{j=0}^{\infty} 2^{j s q}\left|\mathcal{F}^{-1} \varphi_{j} \mathcal{F} f(\cdot)\right|^{q}\right)^{\frac{1}{q}}\right| L_{p}\left(\mathbb{R}^{n}\right)\right\|
$$

(with the usual modification if $q=\infty$ ) is finite.

When $b=0$, part (i) of the definition above coincides with the usual definition for $B$-spaces, $B_{p, q}^{(s, 0)}=B_{p, q}^{s}($ see [18: Definition 2.3.1/2, p. 45]).

The theory of the spaces $B_{p, q}^{s}(b=0)$ and $F_{p, q}^{s}$ has been developed in detail in [18, 19]. These two scales $B_{p, q}^{s}$ and $F_{p, q}^{s}$ cover (fractional) Sobolev spaces, Hölder-Zygmund spaces, local Hardy spaces, and classical Besov spaces - characterised via derivatives and differences. Moreover, there are extensions to these concepts leading to spaces of generalised smoothness. They have been studied in great detail by the Russian school, mainly Lizorkin, Gol'dman and Kalyabin. Quite recently Leopold studied spaces of the type $B_{p, q}^{(s, b)}(b \in \mathbb{R})$ as defined in (6) (see [16]). 


\section{Equivalent norms}

We consider spaces of the type $\operatorname{Lip}_{p, q}^{(1,-\alpha)}$ and $B_{p, q}^{(s, b)}$ (see Definitions 1 and 4 ) and give some equivalent characterisations which will be more convenient to handle in the sequel. Let all spaces be defined on $\mathbb{R}^{n}$ unless otherwise stated. Recall that we have in $B$-spaces the equivalent norm

$$
\left\|f\left|B_{p, q}^{s}\|\sim\| f\right| L_{p}\right\|+\left(\int_{0}^{1 / 2}\left[\frac{\omega_{r}(f, t)_{p}}{t^{s}}\right]^{q} \frac{d t}{t}\right)^{\frac{1}{q}}
$$

(with the usual modification if $q=\infty$ ). Here $r \in \mathbb{N}$ is such that $r>s$ (see [3: Chapter 5/Definition 4.3, p. 332], [7: Chapter 2/§10, pp. 54-56] (where the Besov spaces are defined in that way) and [18: Theorem 2.5.12, p. 110] for what concerns the equivalence result).

The following extrapolation type result for spaces $\operatorname{Lip}_{p, \infty}^{(1,-\alpha)}$ is known.

Proposition 5. Let $1 \leq p \leq \infty$ and $\alpha>0$. Then $f \in \operatorname{Lip}_{p, \infty}^{(1,-\alpha)}$ if, and only if, $f$ belongs to $L_{p}$ and there is some $c>0$ such that for all $\lambda$ with $0<\lambda<1$

$$
\sup _{0<t<\frac{1}{2}} \frac{\omega(f, t)_{p}}{t^{1-\lambda}} \leq c \lambda^{-\alpha}
$$

Moreover, we obtain as an equivalent norm in $\operatorname{Lip}_{p, \infty}^{(1,-\alpha)}$

$$
\left\|f\left|\operatorname{Lip}_{p, \infty}^{(1,-\alpha)}\|\sim\| f\right| L_{p}\right\|+\sup _{0<\lambda<1} \lambda^{\alpha} \sup _{0<t<\frac{1}{2}} \frac{\omega(f, t)_{p}}{t^{1-\lambda}} .
$$

Remark 6. Proposition 5 and its proof can be found in [8: Proposition 4.2/(i)]. Note that when $p=\infty$ it coincides with the result of Krbec and Schmeisser in [14: Proposition 2.5].

We want to mention some apparently elegant, but dangerous notation replacing (9). In view of (8) with $r=1$ and $s=1-\lambda, q=\infty$, i.e

$$
\left\|f\left|B_{p, \infty}^{1-\lambda}\|\sim\| f\right| L_{p}\right\|+\sup _{0<t<\frac{1}{2}} \frac{\omega(f, t)_{p}}{t^{1-\lambda}}
$$

one might be tempted to shorten (9) by

$$
\left\|f\left|\operatorname{Lip}_{p, \infty}^{(1,-\alpha)}\left\|\sim \sup _{0<\lambda<1} \lambda^{\alpha}\right\| f\right| B_{p, \infty}^{1-\lambda}\right\|
$$

However, the (hidden) equivalence constants in (10) depend upon $\lambda$, especially for $\lambda \downarrow 0$, thus one either has to calculate this dependence explicitly, or has to note that the $B$ spaces in (11) are defined via first differences only (in contrast to the usual Fourieranalytical approach). Hence we prefer the slightly more complicated but correct formulation of the equivalence (9) not to be misled.

We give the natural counterpart of (9) when dealing with spaces $\operatorname{Lip}_{p, q}^{(1,-\alpha)} \quad(1 \leq$ $\left.p \leq \infty, 0<q<\infty, \alpha>\frac{1}{q}\right)$. 
Proposition 7. Let $1 \leq p \leq \infty, 0<q<\infty$ and $\alpha>\frac{1}{q}$. Then $f \in \operatorname{Lip}_{p, q}^{(1,-\alpha)}$ if, and only if, $f$ belongs to $L_{p}$ and there is some $c>0$ such that

$$
\int_{0}^{1} \lambda^{\alpha q} \int_{0}^{1 / 2}\left[\frac{\omega(f, t)_{p}}{t^{1-\lambda}}\right]^{q} \frac{d t}{t} \frac{d \lambda}{\lambda} \leq c
$$

Moreover,

$$
\left\|f\left|\operatorname{Lip}_{p, q}^{(1,-\alpha)}\|\sim\| f\right| L_{p}\right\|+\left(\int_{0}^{1} \lambda^{\alpha q} \int_{0}^{1 / 2}\left[\frac{\omega(f, t)_{p}}{t^{1-\lambda}}\right]^{q} \frac{d t}{t} \frac{d \lambda}{\lambda}\right)^{\frac{1}{q}} .
$$

Note that the results (9) and (12) as well as the proof below resemble in some sense the argument given in [10: Section 2.6.2, pp. 69-71] concerning the spaces $L_{p}(\log L)_{a} \quad(1<p<\infty, a \in \mathbb{R})$. Furthermore, in the sense of the above Remark 6 it seems an obvious but dangerous notation in our opinion to replace (12) by

$$
\left\|f \mid \operatorname{Lip}_{p, q}^{(1,-\alpha)}\right\| \sim\left(\int_{0}^{1} \lambda^{\alpha q}\left\|f \mid B_{p, q}^{1-\lambda}\right\|^{q} \frac{d \lambda}{\lambda}\right)^{\frac{1}{q}} .
$$

Strictly spoken, the problems with this notation are the same as described in Remark 6 above (for $q=\infty$ ), we thus stick at (12).

Proof of Proposition 7. In view of (5) it is sufficient to verify that

$$
\int_{0}^{1} \lambda^{\alpha q} \int_{0}^{1 / 2}\left[\frac{\omega(f, t)_{p}}{t^{1-\lambda}}\right]^{q} \frac{d t}{t} \frac{d \lambda}{\lambda} \sim \int_{0}^{1 / 2}\left[\frac{\omega(f, t)_{p}}{t|\log t|^{\alpha}}\right]^{q} \frac{d t}{t} .
$$

Furthermore, by Fubini's theorem this reduces to showing that

$$
\int_{0}^{1} \lambda^{\alpha q-1} t^{\lambda q} d \lambda \sim|\log t|^{-\alpha q} .
$$

Using the substitution $\mu=\lambda q|\log t|$ we arrive at

$$
\int_{0}^{1} \lambda^{\alpha q-1} t^{\lambda q} d \lambda=q^{-\alpha q}|\log t|^{-\alpha q} \int_{0}^{q|\log t|} \mu^{\alpha q-1} e^{-\mu} d \mu
$$

The last term in (14) tends to $\Gamma(\alpha q)$ when $t \downarrow 0$. Thus (13) is shown (recall $0<t<\frac{1}{2}$ )

We come to some counterpart of (8) when dealing with spaces of type $B_{p, q}^{(s, b)}(b \in \mathbb{R})$.

Proposition 8. Let $1 \leq p \leq \infty, 0<q \leq \infty$ and $b \geq 0$. Then

$$
\left\|f\left|B_{p, q}^{(1,-b)}\|\sim\| f\right| L_{p}\right\|+\left(\int_{0}^{1 / 2}\left[\frac{\omega_{2}(f, t)_{p}}{t|\log t|^{b}}\right]^{q} \frac{d t}{t}\right)^{\frac{1}{q}}
$$

(with the usual modification for $q=\infty$ ).

Proof. Note that (15) coincides with (8) for $b=0, s=1$ and $r=2$. The proof consists of suitable adaptions of the related proofs for $b=0$ in [18], that is Theorem 2.5.12/(i) in [18: pp. 110/111], and subsequently [18: Theorem 2.3.6/(i), p.56] and [18: Theorem 2.5.3/(i), pp. $80-83]$. The necessary modifications are obvious 
Remark 9. In view of [18: Theorem 2.5.12/(i)] one can extend (15) to spaces $B_{p, q}^{(s, b)}$ with $0<p \leq \infty, s>n\left(\frac{1}{p}-1\right)_{+}, b \in \mathbb{R}$ and $0<q \leq \infty$, where $\omega_{2}(f, t)_{p}$ has to be replaced by $\omega_{r}(f, t)_{p}$ with $r>s \quad(r \in \mathbb{N})$,

$$
\left\|f\left|B_{p, q}^{(s,-b)}\|\sim\| f\right| L_{p}\right\|+\left(\int_{0}^{1 / 2}\left[\frac{\omega_{r}(f, t)_{p}}{t^{s}|\log t|^{b}}\right]^{q} \frac{d t}{t}\right)^{\frac{1}{q}}<\infty .
$$

In particular, for $p=q=\infty$ we arrive at spaces of Zygmund type, $\mathcal{C}^{(s,-\alpha)}=B_{\infty, \infty}^{(s,-\alpha)}$ $(s>0, \alpha \in \mathbb{R})$,

$$
\left\|f\left|\mathcal{C}^{(s,-\alpha)}\|=\| f\right| L_{\infty}\right\|+\sup _{0<t<\frac{1}{2}} \frac{\omega_{r}(f, t)}{t^{s}|\log t|^{\alpha}}
$$

where $r \in \mathbb{N}$ with $r>s$.

\section{Embeddings}

Recall that all spaces are defined on $\mathbb{R}^{n}$ unless otherwise stated.

3.1 Embeddings into spaces of Lipschitz type. In [8: Proposition 4.2/(ii)] we achieved the following result.

Proposition 10. Let $1 \leq p \leq \infty, 0<q \leq \infty$ and $\alpha>0$. Then

$$
B_{p, q}^{1} \hookrightarrow \operatorname{Lip}_{p, \infty}^{(1,-\alpha)} \quad \text { if } \alpha \geq \frac{1}{q^{\prime}} .
$$

Note that in the case of $p=\infty$ one recovers in that way (a weaker version of) the embedding theorem [9: Theorem 2.1/(ii)]. The counterpart of (17) for spaces $\operatorname{Lip}_{p, q}^{(1,-\alpha)}$ reads as follows.

Proposition 11. Let $1 \leq p \leq \infty, 0<q, v \leq \infty$ and $\alpha>\frac{1}{v}$. Then

$$
B_{p, q}^{1} \hookrightarrow \operatorname{Lip}_{p, v}^{(1,-\alpha)} \quad \text { if }\left\{\begin{array}{lll}
\alpha \geq \frac{1}{q^{\prime}} & \text { and } & v=\infty \\
\alpha>\frac{1}{v}+\frac{1}{q^{\prime}} & \text { and } & v<\infty .
\end{array}\right.
$$

Proof. The upper line in (18) is covered by (17), thus we assume $v<\infty$. In view of (5) and (8) it is sufficient to show that

$$
\left(\int_{0}^{1 / 2}\left[\frac{\omega(f, t)_{p}}{t|\log t|^{\alpha}}\right]^{v} \frac{d t}{t}\right)^{\frac{1}{v}} \leq c\left\|f \mid B_{p, q}^{1}\right\|
$$

if $\alpha>\frac{1}{v}+\frac{1}{q^{\prime}}$. But obviously

$$
\begin{aligned}
\left(\int_{0}^{1 / 2}\left[\frac{\omega(f, t)_{p}}{t|\log t|^{\alpha}}\right]^{v} \frac{d t}{t}\right)^{\frac{1}{v}} & \leq \sup _{0<t<\frac{1}{2}} \frac{\omega(f, t)_{p}}{t|\log t|^{\frac{1}{q^{\prime}}}}\left(\int_{0}^{1 / 2}|\log t|^{-\left(\alpha-\frac{1}{q^{\prime}}\right) v} \frac{d t}{t}\right)^{\frac{1}{v}} \\
& \leq c\left\|f \mid \operatorname{Lip}_{p, \infty}^{(1,-\alpha)}\right\| \\
& \leq c^{\prime}\left\|f \mid B_{p, q}^{1}\right\|
\end{aligned}
$$

where we used (5) and $\alpha-\frac{1}{q^{\prime}}>\frac{1}{v}$ in the penultimate inequality. The last estimate is covered by (17). This ends the proof of Proposition 11 
Remark 12. In view of the above proof it is clear that the 'critical case', that is, $\alpha=\frac{1}{q^{\prime}}+\frac{1}{v}$, cannot be handled in the same way, because

$$
\int_{0}^{1 / 2}|\log t|^{-\left(\alpha-\frac{1}{q^{\prime}}\right) v} \frac{d t}{t}
$$

fails to converge then and the whole argument breaks down. However, there is some reason to believe that embedding (18) holds for $\alpha=\frac{1}{q^{\prime}}+\frac{1}{v}$, too, at least when $1 \leq$ $v, q \leq \infty$ : On the one hand, there is the case $v=\infty$ (see (17)) which supports this conjecture. On the other hand, there is a corresponding limiting result by Triebel when $1<v=q \leq \infty$ and $\alpha \geq 1$ (see Remark 14 below).

Note that Proposition 11 can also be proved directly, that is without application of Proposition 10, by application of Marchaud's inequality (see (32) below). We do not know so far whether the 'critical case' $\alpha=\frac{1}{q^{\prime}}+\frac{1}{v}$ in (17) can be obtained by a (refined) version or application of Marchaud's inequality. Some 'weak' point in an argument of that type is always the application of Hölder's inequality where one might lose sharper results already (see Proposition 16 and its proof (especially Step 3) below). So one has probably to strengthen different techniques, like atomic decompositions, say, in order to prove (17) when $\alpha=\frac{1}{q^{\prime}}+\frac{1}{v}$. Note that our original proof of (17) (with $p=\infty$ ) in [9] is based on an atomic decomposition, too.

Let us finally mention that, at least for $p=\infty$, it is easy to see that $\alpha=\frac{1}{q^{\prime}}+\frac{1}{v}$ is in fact the best, that is the smallest possible log-exponent in (17). In other words, the embedding fails when $\alpha<\frac{1}{q^{\prime}}+\frac{1}{v}$. This becomes evident in particular in the next section, we thus postpone the argument to Remark 19 below.

The most interesting result is certainly the case $v=q$ and $p=\infty$ in (18). Additionally, involving some elementary embeddings for $B_{p, q}^{s}$ spaces we have proved the following.

Corollary 13. Let $0<p \leq \infty, 0<q \leq \infty$ and $\alpha>\frac{1}{q}$. Then

$$
B_{p, q}^{1+\frac{n}{p}} \hookrightarrow \operatorname{Lip}_{\infty, q}^{(1,-\alpha)} \quad \text { if }\left\{\begin{array}{lll}
\alpha \geq 1 & \text { and } & q=\infty, \\
\alpha>\max \left(1, \frac{1}{q}\right) & \text { and } & 0<q<\infty .
\end{array}\right.
$$

Remark 14. Triebel proved in some so far unpublished notes that (19) holds with $\alpha=1$ when $1<q \leq \infty$ and $0<p<\infty$, using different techniques (involving nonincreasing rearrangement, Hardy inequalities and atomic decompositions).

We briefly turn to $F$-spaces. Recall the following result first.

Proposition 15. Let $0<p<\infty, 0<q \leq \infty$ and $\alpha \geq 0$. Then

$$
F_{p, q}^{1+\frac{n}{p}} \hookrightarrow \operatorname{Lip}_{\infty, \infty}^{(1,-\alpha)} \quad \text { if } \alpha \geq \frac{1}{p^{\prime}}
$$

We proved this assertion in [9: Theorem 2.1/(i)]. Moreover, the exponent $\alpha=\frac{1}{p^{\prime}}$, when $1<p<\infty$, is sharp. Note that in the case of $1<p<\infty$ and $q=2(20)$ reproduces the famous Brézis-Wainger result [6]. 
There is also an extension of Proposition 15 to spaces $\operatorname{Lip}_{p, q}^{(1,-\alpha)}$, but is is more convenient for us to postpone this result to Corollary 20 below.

3.2 Embeddings of purely Lipschitzian type. We study embeddings now where the initial space as well as the target one is of type $\operatorname{Lip}_{p, q}^{(1,-\alpha)}$.

Proposition 16. Let $1 \leq p \leq \infty, 0<q, v \leq \infty$ and $\alpha>\frac{1}{q}, \beta>\frac{1}{v}$. Then

$$
\operatorname{Lip}_{p, q}^{(1,-\alpha)} \hookrightarrow \operatorname{Lip}_{p, v}^{(1,-\beta)} \quad \text { if, and only if, }\left\{\begin{array}{l}
\beta-\frac{1}{v} \geq \alpha-\frac{1}{q} \quad \text { and } \quad v \geq q \\
\beta-\frac{1}{v}>\alpha-\frac{1}{q} \quad \text { and } \quad v<q
\end{array}\right.
$$

Proof. Note that the upper line in (21) is somehow surprising, as it means that some space $\operatorname{Lip}_{p, q}^{(1,-\alpha)}$ can be continuously embedded into $\operatorname{Lip}_{p, v}^{(1,-\beta)}$ even if $\beta<\alpha$. At first glance this seems impossible: having 'less' (logarithmic) smoothness $(-\alpha)$ in the original space than in the target one $(-\beta)$; but it turns out that this fact simply refers back to the influence of $q$ in Definition 1. The argument to prove it is indeed a tricky one and due to Bennett and Rudnick in [2] (as far as we know) - what they call some 'diagonal' result. But we return to this point later in the proof.

Step 1. We first prove the necessity of the assumptions on the parameters in (21). Let $f_{\kappa, \mu} \in L_{p}$ be such that

$$
\omega\left(f_{\kappa, \mu}, t\right)_{p} \sim t|\log t|^{\kappa}|\log | \log t||^{-\mu}
$$

for small $t>0$ and $\kappa, \mu \in \mathbb{R}$. Note that $f_{\kappa, 0} \in \operatorname{Lip}_{p, q}^{(1,-\alpha)}$ if, and only if, $\kappa<\alpha-\frac{1}{q}$. Thus, assuming $\beta-\frac{1}{v}<\alpha-\frac{1}{q}$ and $0<q, v \leq \infty$, we may choose $\kappa$ such that $\beta-\frac{1}{v} \leq \kappa<\alpha-\frac{1}{q}$ and hence $f_{\kappa, 0} \in \operatorname{Lip}_{p, q}^{(1,-\alpha)} \backslash \operatorname{Lip}_{p, v}^{(1,-\beta)}$. Now let $\beta-\frac{1}{v}=\alpha-\frac{1}{q}=: \kappa$. Then $f_{\kappa, \mu} \in \operatorname{Lip}_{p, q}^{(1,-\alpha)}$ if, and only if, $\mu>\frac{1}{q}$. Consequently, $f_{\kappa, \mu} \in \operatorname{Lip}_{p, q}^{(1,-\alpha)} \backslash \operatorname{Lip}_{p, v}^{(1,-\beta)}$ if $\kappa=\beta-\frac{1}{v}=\alpha-\frac{1}{q}$ and $\frac{1}{q}<\mu \leq \frac{1}{v}$, that is, when $\beta-\frac{1}{v}=\alpha-\frac{1}{q}$ and $v<q$.

Step 2. We prove the sufficiency in (21) in case of $v<q$. In view of (5) we have to show that

$$
\left(\int_{0}^{1 / 2}\left[\frac{\omega(f, t)_{p}}{t|\log t|^{\beta}}\right]^{v} \frac{d t}{t}\right)^{\frac{1}{v}} \leq c\left(\int_{0}^{1 / 2}\left[\frac{\omega(f, t)_{p}}{t|\log t|^{\alpha}}\right]^{q} \frac{d t}{t}\right)^{\frac{1}{q}} .
$$

By Hölder's inequality we get

$$
\begin{aligned}
& \left(\int_{0}^{1 / 2}\left[\frac{\omega(f, t)_{p}}{t|\log t|^{\beta}}\right]^{v} \frac{d t}{t}\right)^{\frac{1}{v}} \\
& \quad \leq c\left(\int_{0}^{1 / 2}\left[\frac{\omega(f, t)_{p}}{t|\log t|^{\alpha}}\right]^{q} \frac{d t}{t}\right)^{\frac{1}{q}}\left(\int_{0}^{1 / 2}|\log t|^{-\frac{\beta-\alpha}{1 / v-1 / q}} \frac{d t}{t}\right)^{\frac{1}{v}-\frac{1}{q}} \\
& \quad \leq c^{\prime}\left(\int_{0}^{1 / 2}\left[\frac{\omega(f, t)_{p}}{t|\log t|^{\alpha}}\right]^{q} \frac{d t}{t}\right)^{\frac{1}{q}}
\end{aligned}
$$

where the last estimate is correct for $\beta-\alpha>\frac{1}{v}-\frac{1}{q}$. This gives the lower line in (21). 
Step 3. It remains to verify the upper line in (21). By the obvious monotonicity argument it is sufficient to prove

$$
\operatorname{Lip}_{p, q}^{(1,-\alpha)} \hookrightarrow \operatorname{Lip}_{p, v}^{(1,-\beta)} \quad \text { if } \beta-\frac{1}{v}=\alpha-\frac{1}{q}, v \geq q .
$$

As already mentioned we make use of a clever trick which can be found in [2: Theorem 9.5, p. 33]. Recall $\alpha>\frac{1}{q}$. Obviously,

$$
\left[|\log t|^{-(\alpha q-1)}\right]^{\prime}=\frac{\alpha q-1}{t|\log t|^{\alpha q}} \quad(t>0)
$$

implies

$$
|\log |^{-(\alpha q-1)}=c \int_{0}^{t} \frac{d \tau}{\tau|\log \tau|^{\alpha q}} .
$$

Bennett and Rudnick in [2: Theorem 9.5] then gained from the fact that for some function $f$ its rearrangement $f^{*}$ is decreasing (by definition). In our case we may replace this argument in the following way : by [7: Chapter $2 / \S 6$, p. $41-42]$ one has that $t^{-1} \omega(f, t)_{p}$ is - roughly speaking - decreasing in $t>0$ (up to constants), such that (24) (after multiplying both sides by $\left[t^{-1} \omega(f, t)_{p}\right]^{q}$ and involving the above-described monotonicity) results for $0<t<\frac{1}{2}$ in

$$
\frac{\omega(f, t)_{p}}{t|\log t|^{\alpha-\frac{1}{q}}} \leq c\left(\int_{0}^{t}\left[\frac{\omega(f, \tau)_{p}}{\tau|\log \tau|^{\alpha}}\right]^{q} \frac{d \tau}{\tau}\right)^{\frac{1}{q}} .
$$

Using the decomposition

$$
\left[\frac{\omega(f, t)_{p}}{t|\log t|^{\beta}}\right]^{v}=\left[\frac{\omega(f, t)_{p}}{t|\log t|^{\alpha}}\right]^{q}\left[\frac{\omega(f, t)_{p}}{t|\log t|^{\alpha-1 / q}}\right]^{v-q}
$$

we apply (25) to the last term on the right-hand side of (26) and recall (23). Thus

$$
\begin{aligned}
& \left(\int_{0}^{1 / 2}\left[\frac{\omega(f, t)_{p}}{t|\log t|^{\beta}}\right]^{v} \frac{d t}{t}\right)^{\frac{1}{v}} \\
& \quad \leq c\left\{\int_{0}^{1 / 2}\left[\frac{\omega(f, t)_{p}}{t|\log t|^{\alpha}}\right]^{q}\left(\int_{0}^{1 / 2}\left[\frac{\omega(f, \tau)_{p}}{\tau|\log \tau|^{\alpha}}\right]^{q} \frac{d \tau}{\tau}\right)^{\frac{v-q}{q}} \frac{d t}{t}\right\}^{\frac{1}{v}} \\
& \quad=c\left(\int_{0}^{1 / 2}\left[\frac{\omega(f, \tau)_{p}}{\tau|\log \tau|^{\alpha}}\right]^{q} \frac{d \tau}{\tau}\right)^{\frac{v-q}{v q}}\left\{\int_{0}^{1 / 2}\left[\frac{\omega(f, t)_{p}}{t|\log t|^{\alpha}}\right]^{q} \frac{d t}{t}\right\}^{\frac{1}{v}} \\
& \quad=c\left(\int_{0}^{1 / 2}\left[\frac{\omega(f, t)_{p}}{t|\log t|^{\alpha}}\right]^{q} \frac{d t}{t}\right)^{\frac{1}{q}} .
\end{aligned}
$$

This gives (23) 
Remark 17. One recognises that our result (21) resembles the outcome [2: Theorems 9.3 and 9.5] by Bennett and Rudnick when in their setting $p=\infty$. We already mentioned the somehow astonishing result that concerning the embedding $\operatorname{Lip}_{p, q}^{(1,-\alpha)}$ into $\operatorname{Lip}_{p, v}^{(1,-\beta)}$ one can 'compensate' some gain of logarithmic smoothness $-\beta>-\alpha$ by 'paying' with the additional index $q$, that is, as long as $(-\beta)-(-\alpha) \leq \frac{1}{q}-\frac{1}{v}, v \geq q$.

This situation is essentially different from the related one when dealing with spaces $B_{p, q}^{(s, b)}$, but we postpone a discussion of this phenomenon to Section 4 .

Remark 18. We used in Step 3 of the above proof that $t^{-1} \omega(f, t)_{p} \quad(1 \leq p \leq \infty)$ is (more or less) decreasing in $t>0$. This fact immediately implies that $\operatorname{Lip}_{p, q}^{(1,-\alpha)}$ with $0<q<\infty$ and $\alpha \leq \frac{1}{q}$ is a very poor space (see Remark 2). For assuming that there is some $c>0$ such that

$$
\frac{\omega(f, t)_{p}}{t} \geq c>0
$$

for small $t>0$, one can estimate

$$
\left\|f \mid \operatorname{Lip}_{p, q}^{(1,-\alpha)}\right\| \geq C\left(\int_{0}^{1 / 2} \frac{1}{|\log t|^{\alpha q}} \frac{d t}{t}\right)^{\frac{1}{q}}
$$

but the right-hand side diverges for $\alpha \leq \frac{1}{q}$. Thus, conversely, for a function $f \in$ $\operatorname{Lip}_{p, q}^{(1,-\alpha)}\left(0<q<\infty, \alpha \leq \frac{1}{q}\right)$ we have to assume $\omega(f, t)_{p}=0$ for small $t>0-$ and the only constant belonging to $L_{p}$ is the null function.

Remark 19. We show that the embedding $B_{p, q}^{1} \hookrightarrow \operatorname{Lip}_{p, v}^{(1,-\alpha)}$ fails for $\alpha<\frac{1}{q^{\prime}}+\frac{1}{v}$. For convenience, let $p=\infty$; but an adapted argument should work for $p<\infty$, too. Assume that $B_{\infty, q}^{1} \hookrightarrow \operatorname{Lip}_{\infty, v}^{(1,-\alpha)}$ for some $\alpha<\frac{1}{q^{\prime}}+\frac{1}{v}$. Then by (21) we may continue

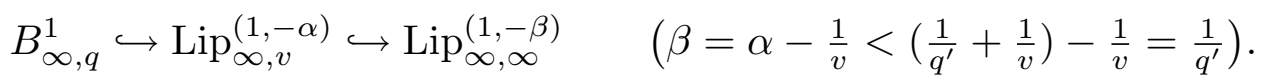

However, this contradicts the sharpness assertion in [9: Theorem 2.1/(ii)] stating that $B_{\infty, q}^{1} \hookrightarrow \operatorname{Lip}_{\infty, \infty}^{(1,-\beta)}$ if, and only if, $\beta \geq \frac{1}{q^{\prime}}$. Hence $B_{p, q}^{1} \hookrightarrow \operatorname{Lip}_{p, v}^{(1,-\alpha)}$ implies $\alpha \geq \frac{1}{q^{\prime}}+\frac{1}{v}$, at least for $p=\infty$. In particular, when $1 \leq q=v \leq \infty$, we necessarily have $\alpha \geq 1$ (see $(19))$.

We now give the promised extension of Proposition 15. Obviously, Proposition 16 and (20) imply the following.

Corollary 20. Let $0<p<\infty, 0<q, v \leq \infty$ and $\alpha>\frac{1}{v}$. Then

$$
F_{p, q}^{1+\frac{n}{p}} \hookrightarrow \operatorname{Lip}_{\infty, v}^{(1,-\alpha)} \quad \text { if }\left\{\begin{array}{lll}
\alpha \geq \frac{1}{p^{\prime}} & \text { and } & v=\infty \\
\alpha>\frac{1}{v}+\frac{1}{p^{\prime}} & \text { and } & v<\infty
\end{array}\right.
$$

In particular,

$$
F_{p, q}^{1+\frac{n}{p}} \hookrightarrow \operatorname{Lip}_{\infty, p}^{(1,-\alpha)} \quad \text { if } \alpha>\max \left(1, \frac{1}{p}\right)
$$


Remark 21. Parallel to Remark 14 we mention that Triebel obtained instead of (27) a sharper assertion with $\alpha=1$, when $1<p<\infty$. Note that by a similar argument, i.e. combination of (17) and (21), we get an alternative proof of Proposition 11.

3.3 Embeddings between 'logarithmically smooth' spaces. In Section 4 we intend to compare spaces of the types $B_{p, q}^{(s, b)}$ and $\operatorname{Lip}_{p, q}^{(1,-\alpha)}$ in some detail. Thus it is of particular interest to derive a few more, rather elementary embeddings between both scales of spaces. We studied this question for $p=q=\infty$ in [9: Proposition 4.2] and obtained the following.

Proposition 22. Let $\alpha \geq 0$. Then

$$
B_{\infty, 1}^{(1,-\alpha)} \hookrightarrow \operatorname{Lip}_{\infty, \infty}^{(1,-\alpha)} \hookrightarrow B_{\infty, \infty}^{(1,-\alpha)} .
$$

Moreover, we proved in [9: Proposition 4.4] that

$$
B_{\infty, q}^{(1,-\alpha)} \hookrightarrow \operatorname{Lip}_{\infty, \infty}^{(1,-\alpha)} \quad \text { if, and only if, } 0<q \leq 1 .
$$

In view of characterisation (15) and Marchaud's inequality we may extend Proposition 22 to spaces $\operatorname{Lip}_{p, q}^{(1,-\alpha)}$.

Corollary 23. Let $1 \leq p \leq \infty, 0<q \leq \infty$ and $\alpha>\frac{1}{q}$.

(i) Then

$$
B_{p, 1}^{(1,-\beta)} \hookrightarrow \operatorname{Lip}_{p, q}^{(1,-\alpha)} \quad \text { if } \begin{cases}\beta<\alpha-\frac{1}{q} & \text { and } 0<q<\infty \\ \beta \leq \alpha & \text { and } q=\infty\end{cases}
$$

Moreover,

$$
B_{p, \min (q, 1)}^{\left(1,-\left(\alpha-\frac{1}{q}\right)\right)} \hookrightarrow \operatorname{Lip}_{p, q}^{(1,-\alpha)}
$$

(ii) Then

$$
\operatorname{Lip}_{p, q}^{(1,-\alpha)} \hookrightarrow B_{p, \infty}^{\left(1,-\left(\alpha-\frac{1}{q}\right)\right)}
$$

\section{Proof.}

Step 1. We deal with (28). Note that for $q \geq 1$ (28) is a consequence of (29), but we will prove both assertions separately. In view of (15) and (5) it is sufficient to show that

$$
\left(\int_{0}^{1 / 2}\left[\frac{\omega(f, t)_{p}}{t|\log t|^{\alpha}}\right]^{q} \frac{d t}{t}\right)^{\frac{1}{q}} \leq c\left\{\left\|f \mid L_{p}\right\|+\int_{0}^{1 / 2} \frac{\omega_{2}(f, t)_{p}}{t|\log t|^{\beta}} \frac{d t}{t}\right\}
$$

where $\beta<\alpha-\frac{1}{q}$ and $0<q<\infty$ (the modifications for $q=\infty$ will be clear from the argument below). Furthermore, without restriction of generality we can assume that $0 \leq$ $\beta<\alpha-\frac{1}{q}$, the rest is done by the monotonicity of $B_{p, q}^{(s, b)}$ spaces. We apply Marchaud's inequality (see [3: Chapter 5/Formula (4.11), p. 334] or [7: Chapter 2/Theorem 8.1, p. 47]) which states the following : let $f \in L_{p} \quad(1 \leq p \leq \infty), t>0$ and $k \in \mathbb{N}$. Then

$$
\omega_{k}(f, t)_{p} \leq \frac{k}{\log 2} t^{k} \int_{t}^{\infty} \frac{\omega_{k+1}(f, u)_{p}}{u^{k}} \frac{d u}{u} .
$$


In particular, assuming $k=1$, then (32) implies that there is some $c>0$ such that

$$
\omega(f, t)_{p} \leq c t \int_{t}^{\infty} \frac{\omega_{2}(f, u)_{p}}{u} \frac{d u}{u}
$$

for all $f \in L_{p}$ and $t>0$. We thus may conclude that

$$
\begin{aligned}
& \left(\int_{0}^{1 / 2}\left[\frac{\omega(f, t)_{p}}{t|\log t|^{\alpha}}\right]^{q} \frac{d t}{t}\right)^{\frac{1}{q}} \\
& \quad=\left(\int_{0}^{1 / 2} \frac{1}{|\log t|^{\alpha q}}\left[\frac{\omega(f, t)_{p}}{t}\right]^{q} \frac{d t}{t}\right)^{\frac{1}{q}} \\
& \quad \leq c\left\{\left\|f \mid L_{p}\right\|+\left(\int_{0}^{1 / 2} \frac{1}{|\log t|^{\alpha q}}\left[\int_{t}^{1 / 2} \frac{\omega_{2}(f, u)_{p}}{u} \frac{d u}{u}\right]^{q} \frac{d t}{t}\right)^{\frac{1}{q}}\right\} .
\end{aligned}
$$

By the monotonicity of the log-function we have

$$
\int_{t}^{1 / 2} \frac{\omega_{2}(f, u)_{p}}{u} \frac{d u}{u} \leq c|\log t|^{\beta} \int_{t}^{1 / 2} \frac{\omega_{2}(f, u)_{p}}{u|\log u|^{\beta}} \frac{d u}{u} \leq c|\log t|^{\beta} \int_{0}^{1 / 2} \frac{\omega_{2}(f, u)_{p}}{u|\log u|^{\beta}} \frac{d u}{u}
$$

for any $t$ with $0<t<\frac{1}{2}$; recall $\beta \geq 0$. Thus (33) implies

$$
\begin{aligned}
& \left(\int_{0}^{1 / 2}\left[\frac{\omega(f, t)_{p}}{t|\log t|^{\alpha}}\right]^{q} \frac{d t}{t}\right)^{\frac{1}{q}} \\
& \quad \leq c\left\{\left\|f \mid L_{p}\right\|+\left(\int_{0}^{1 / 2} \frac{1}{|\log t|^{(\alpha-\beta) q}} \frac{d t}{t}\right)^{\frac{1}{q}}\left[\int_{0}^{1 / 2} \frac{\omega_{2}(f, u)_{p}}{u|\log u|^{\beta}} \frac{d u}{u}\right]\right\} \\
& \quad \leq c^{\prime}\left\|f \mid B_{p, 1}^{(1,-\beta)}\right\|
\end{aligned}
$$

where the last inequality is correct for $\beta<\alpha-\frac{1}{q}$. In case of $q=\infty$ one has to modify the above argument in an obvious manner. Hence (28) is shown.

Step 2. We prove (29). Let first $0<q \leq 1$. Then (31) has to be replaced by

$$
\left(\int_{0}^{1 / 2}\left[\frac{\omega(f, t)_{p}}{t|\log t|^{\alpha}}\right]^{q} \frac{d t}{t}\right)^{\frac{1}{q}} \leq c\left\{\left\|f \mid L_{p}\right\|+\left(\int_{0}^{1 / 2}\left[\frac{\omega_{2}(f, t)_{p}}{t|\log t|^{\alpha-\frac{1}{q}}}\right]^{q} \frac{d t}{t}\right)^{\frac{1}{q}}\right\} .
$$

Recall $0<q \leq 1$, thus (33) and Fubini's theorem yield

$$
\begin{gathered}
\left(\int_{0}^{1 / 2}\left[\frac{\omega(f, t)_{p}}{t|\log t|^{\alpha}}\right]^{q} \frac{d t}{t}\right)^{\frac{1}{q}} \\
\quad \leq c\left\{\left\|f \mid L_{p}\right\|+\left(\int_{0}^{1 / 2} \frac{1}{|\log t|^{\alpha q}} \int_{t}^{1 / 2}\left[\frac{\omega_{2}(f, u)_{p}}{u}\right]^{q} \frac{d u}{u} \frac{d t}{t}\right)^{\frac{1}{q}}\right\} \\
\quad=c\left\{\left\|f \mid L_{p}\right\|+\left(\int_{0}^{1 / 2}\left[\frac{\omega_{2}(f, u)_{p}}{u}\right]^{q} \int_{0}^{u} \frac{1}{|\log t|^{\alpha q}} \frac{d t}{t} \frac{d u}{u}\right)^{\frac{1}{q}}\right\} \\
\leq c^{\prime}\left\{\left\|f \mid L_{p}\right\|+\left(\int_{0}^{1 / 2}\left[\frac{\omega_{2}(f, u)_{p}}{u|\log u|^{\alpha-\frac{1}{q}}}\right]^{q} \frac{d u}{u}\right)^{\frac{1}{q}}\right\}
\end{gathered}
$$


where we also used $\alpha>\frac{1}{q}$. This gives (34), i.e. (29) for $0<q \leq 1$. Assume now $1 \leq q \leq \infty$. Put $\beta:=1+\left(\alpha-\frac{1}{q}\right)>1$, i.e. $\alpha-\frac{1}{q}=\beta-1$. The just proved result (29) with $q=1$ implies

$$
B_{p, 1}^{\left(1,-\left(\alpha-\frac{1}{q}\right)\right)}=B_{p, 1}^{(1,-(\beta-1))} \hookrightarrow \operatorname{Lip}_{p, 1}^{(1,-\beta)} .
$$

Moreover, (21) provides $\operatorname{Lip}_{p, 1}^{(1,-\beta)} \hookrightarrow \operatorname{Lip}_{p, q}^{(1,-\alpha)}$ because $q \geq 1$ and $\beta-1=\alpha-\frac{1}{q}$. Thus we can continue (35) to the desired result (29), now for $1 \leq q \leq \infty$.

Step 3. It remains to show (30). We gain from Proposition 16 in the following way: by (21) we have $\operatorname{Lip}_{p, q}^{(1,-\alpha)} \hookrightarrow \operatorname{Lip}_{p, \infty}^{\left(1,-\left(\alpha-\frac{1}{q}\right)\right)}$ and can thus reduce (30) to the verification of $\operatorname{Lip}_{p, \infty}^{(1,-\gamma)} \hookrightarrow B_{p, \infty}^{(1,-\gamma)}$ for some $\gamma>0$ and $1 \leq p \leq \infty$. But this is obvious by (15), (5) and $\omega_{2}(f, t)_{p} \leq c \omega(f, t)_{p}$

Remark 24. Note that for $p=q=\infty$ assertions (28) - (30) coincide with [9: Proposition 4.2].

Moreover, by Step 3 of the above proof (in particular, $\operatorname{Lip}_{p, v}^{(1,-\beta)} \hookrightarrow B_{p, v}^{(1,-\beta)}$ for $\beta>\frac{1}{v}$ ) in connection with Proposition 16 we immediately obtain the following extension of (30).

Corollary 25. Let $1 \leq p \leq \infty, 0<q, v \leq \infty$ and $\alpha>\frac{1}{q}, \beta>\frac{1}{v}$. Then

$$
\operatorname{Lip}_{p, q}^{(1,-\alpha)} \hookrightarrow B_{p, v}^{(1,-\beta)} \quad \text { if }\left\{\begin{array}{lll}
\beta-\frac{1}{v} \geq \alpha-\frac{1}{q} & \text { and } \quad v \geq q, \\
\beta-\frac{1}{v}>\alpha-\frac{1}{q} & \text { and } \quad v<q .
\end{array}\right.
$$

On the other hand, we may also complement (29) by a similar assertion.

Corollary 26. Let $1 \leq p \leq \infty, 1 \leq q \leq \infty$ and $\alpha>1$. Then

$$
B_{p, q}^{(1,-(\alpha-1))} \hookrightarrow \operatorname{Lip}_{p, q}^{(1,-\alpha)} .
$$

Proof. We proceed similarly to the proof of Corollary 23. Now (31) has to be replaced by

$$
\left(\int_{0}^{1 / 2}\left[\frac{\omega(f, t)_{p}}{t|\log t|^{\alpha}}\right]^{q} \frac{d t}{t}\right)^{\frac{1}{q}} \leq c\left\{\left\|f \mid L_{p}\right\|+\left(\int_{0}^{1 / 2}\left[\frac{\omega_{2}(f, t)_{p}}{t|\log t|^{\alpha-1}}\right]^{q} \frac{d t}{t}\right)^{\frac{1}{q}}\right\} .
$$

Recall $q \geq 1$, thus (33) together with Hölder's inequality and Fubini's theorem imply

$$
\begin{aligned}
& \left(\int_{0}^{1 / 2}\left[\frac{\omega(f, t)_{p}}{t|\log t|^{\alpha}}\right]^{q} \frac{d t}{t}\right)^{\frac{1}{q}} \\
& \quad \leq c\left\{\left\|f \mid L_{p}\right\|+\left(\int_{0}^{1 / 2} \frac{1}{|\log t|^{q\left(\alpha-\frac{1}{q^{\prime}}\right)}} \int_{t}^{1 / 2}\left[\frac{\omega_{2}(f, u)_{p}}{u}\right]^{q} \frac{d u}{u} \frac{d t}{t}\right)^{\frac{1}{q}}\right\} \\
& \quad=c\left\{\left\|f \mid L_{p}\right\|+\left(\int_{0}^{1 / 2}\left[\frac{\omega_{2}(f, u)_{p}}{u}\right]^{q} \int_{0}^{u} \frac{1}{|\log t|^{q\left(\alpha-\frac{1}{q^{\prime}}\right)}} \frac{d t}{t} \frac{d u}{u}\right)^{\frac{1}{q}}\right\} \\
& \quad \leq c^{\prime}\left\{\left\|f \mid L_{p}\right\|+\left(\int_{0}^{1 / 2}\left[\frac{\omega_{2}(f, u)_{p}}{u|\log u|^{\alpha-1}}\right]^{q} \frac{d u}{u}\right)^{\frac{1}{q}}\right\}
\end{aligned}
$$

because $\alpha>1$. Hence (38) is verified 
Recall the notation for spaces $\mathcal{C}^{(1,-\alpha)} \quad(\alpha \geq 0)$, see (16) with $s=1$ and $r=2$. We proved in [8: Proposition 2.7] that

$$
\operatorname{Lip}_{\infty, \infty}^{(1,-\alpha)} \hookrightarrow \mathcal{C}^{(1,-\beta)} \hookrightarrow \operatorname{Lip}_{\infty, \infty}^{(1,-\gamma)}
$$

if, and only if,

$$
\beta \geq \alpha \quad \text { and } \quad \gamma \geq \beta+1 .
$$

Hence (37) coincides with the right-hand embedding in (39) when $p=q=\infty$.

\section{Some discussion}

We compare 'logarithmically smooth' Besov spaces $B_{p, q}^{(s, b)}$, introduced by Leopold in [16], and 'logarithmic' Lipschitz spaces $\operatorname{Lip}_{p, q}^{(1,-\alpha)}$. From the point of dealing with these spaces in view of atomic decompositions etc., it is essential that the logarithmic $B$-spaces, that is $B_{p, q}^{(s, b)}$ arise by a Fourier-analytical approach (like the usual spaces $B_{p, q}^{s}$; see (6)), whereas the logarithmic Lipschitz spaces $\operatorname{Lip}_{p, q}^{(1,-\alpha)}$, defined via first differences (see (5)), remain as 'Fourier-unfriendly' as were their classical forerunners (with $p=q=\infty$ and $\alpha=0$ ). In fact, the almost inconspicuous modification in (5) compared with (15), namely the substitution of $\omega_{2}(f, t)_{p}$ by $\omega_{1}(f, t)_{p}$, causes a striking difference in the features of the corresponding spaces (as it does for $\alpha=b=0$ ).

We return to Proposition 16 . The counterpart for spaces $B_{p, q}^{(s, b)}$, obtained by Leopold in [16: Theorem 1], reads as follows :

$$
\begin{aligned}
& \text { Let } s \in \mathbb{R}, b_{1}, b_{2} \in \mathbb{R}, 0<p \leq \infty \text { and } 0<q_{1}, q_{2} \leq \infty \text {. Then } \\
& \begin{array}{l}
B_{p, q_{1}}^{\left(s, b_{1}\right)} \hookrightarrow B_{p, q_{2}}^{\left(s, b_{2}\right)} \\
\text { if, and only if, }\left\{\begin{array}{l}
b_{1}-b_{2} \geq 0 \\
b_{1}-b_{2}>\frac{1}{q_{2}}-\frac{1}{q_{1}}
\end{array} \quad \text { and } \quad \begin{array}{l}
q_{1} \leq q_{2}, \\
q_{1}>q_{2} .
\end{array}\right.
\end{array}
\end{aligned}
$$

It is obvious, that - though (21) and (40) appear related somehow - the role played by the parameter $q$ in either case is obviously different. The 'diagonal argument' (essentially used in Step 3 of the proof of Proposition 16 and borrowed from Bennett and Rudnick) does not apply in that case. In other words, the parallel notation (taking the same parameter $q$ ) in both cases $B_{p, q}^{(s, b)}$ and $\operatorname{Lip}_{p, q}^{(1,-\alpha)}$, respectively, is a dangerous one (though suggestive in either case), possibly pretending at first glance that the construction involving $q$ might be the same; however, it is not. On the other hand, it is nevertheless surprising that the 'fine index' $q$ in these limiting cases becomes so important.

Furthermore, we study the question now 'where' the Lipschitz spaces $\operatorname{Lip}_{p, q}^{(1,-\alpha)}$ can be found within the scale of Besov spaces $B_{p, q}^{(s, b)}$. Let $1 \leq p \leq \infty$ and $0<q \leq \infty$. Concerning the scale of logarithmic Besov spaces $B_{p, q}^{(1, b)}$ for fixed $p$ and $q$, but arbitrary $b \in \mathbb{R}$, we may locate the Lipschitz spaces $\operatorname{Lip}_{p, q}^{(1,-\alpha)}$ as follows. Denote by $q^{*}:=\min (q, 1)$ and assume $\alpha>\frac{1}{q^{*}}$. Then

$$
B_{p, q}^{\left(1,-\left(\alpha-\frac{1}{q^{*}}\right)\right)} \hookrightarrow \operatorname{Lip}_{p, q}^{(1,-\alpha)} \hookrightarrow B_{p, q}^{(1,-\alpha)}
$$


(see (29), (36) and (37)). Insisting, however, on the same (logarithmic) smoothness in both nestling spaces of type $B_{p, q}^{(1, b)}$, that is, for fixed $p$ and $b$ but varying $q$, we found

$$
B_{p, q^{*}}^{\left(1,-\left(\alpha-\frac{1}{q}\right)\right)} \hookrightarrow \operatorname{Lip}_{p, q}^{(1,-\alpha)} \hookrightarrow B_{p, \infty}^{\left(1,-\left(\alpha-\frac{1}{q}\right)\right)}
$$

(recall (29) and (30)). Note, finally, that for $1<q<\infty$ the respective initial spaces and endpoint spaces in (41) and (42) are incomparable in the sense that neither of them is contained in the corresponding other one; this refers to $B_{p, q}^{\left(1,-\left(\alpha-\frac{1}{q^{*}}\right)\right)}$ and $B_{p, q^{*}}^{\left(1,-\left(\alpha-\frac{1}{q}\right)\right)}$ as well as to $B_{p, q}^{(1,-\alpha)}$ and $B_{p, \infty}^{\left(1,-\left(\alpha-\frac{1}{q}\right)\right)}$, respectively. Obviously they coincide, respectively, when $0<q \leq 1$ (in the case of the initial spaces) and when $q=\infty$ (concerning the endpoint spaces). Thus we have the general situation that

Recall that we have the same diagram with $\operatorname{Lip}_{p, q}^{(1,-\alpha)}$ replaced by $B_{p, q}^{\left(1,-\left(\alpha-\frac{1}{q}\right)\right)}$. These spaces, however, are not comparable (in the above sense) when $1<q<\infty$. On the one hand, one might strengthen structural arguments to disprove this assumption, but on the other hand it can also be seen as follows. Assume that $\operatorname{Lip}_{p, q}^{(1,-\alpha)} \hookrightarrow B_{p, q}^{\left(1,-\left(\alpha-\frac{1}{q}\right)\right)}$ for some $q<\infty$. But functions of the type $f_{\kappa, 0}$ given by (22) belong to $\operatorname{Lip}_{p, q}^{(1,-\alpha)} \backslash B_{p, q}^{\left(1,-\left(\alpha-\frac{1}{q}\right)\right)}$ choosing $\kappa$ such that $\left(\alpha-\frac{1}{q}\right)-\frac{1}{q}<\kappa<\left(\alpha-\frac{1}{q}\right)$. Conversely, assume that $B_{p, q}^{\left(1,-\left(\alpha-\frac{1}{q}\right)\right)} \hookrightarrow$ $\operatorname{Lip}_{p, q}^{(1,-\alpha)}$ for some $q>1$. Then one can easily disprove this assertion for $p=\infty$ by the following argument: choose $u>q$, thus (40) and (21) imply that

$$
B_{\infty, u}^{1} \hookrightarrow B_{\infty, q}^{\left(1,-\left(\alpha-\frac{1}{q}\right)\right)} \hookrightarrow \operatorname{Lip}_{\infty, q}^{(1,-\alpha)} \hookrightarrow \operatorname{Lip}_{\infty, \infty}^{\left(1,-\left(\alpha-\frac{1}{q}\right)\right)}
$$

for $\alpha-\frac{1}{q}>\frac{1}{q}-\frac{1}{u}$. However, studying the embedding $B_{\infty, u}^{1} \hookrightarrow \operatorname{Lip}_{\infty, \infty}^{\left(1,-\left(\alpha-\frac{1}{q}\right)\right)}$ we obtained in [9: Theorem 2.1/(ii)] that the exponent $\alpha-\frac{1}{q}=1-\frac{1}{u}$ is sharp (see also (17)); but $1-\frac{1}{u}>\frac{1}{q}-\frac{1}{u}$ for $q>1$. Thus $B_{p, q}^{\left(1,-\left(\alpha-\frac{1}{q}\right)\right)}$ cannot be contained in $\operatorname{Lip}_{p, q}^{(1,-\alpha)}$ for $q>1$ (at least when $p=\infty$, but there should be a similar counter-argument when $1 \leq p<\infty$ ). Hence the above diagram (43) looks in general like

where the left part of (44) collapses to one space for $0<q \leq 1$ and likewise the right one when $q=\infty$.

There are a lot of further related approaches to spaces of Lipschitz type (recall Remark 3). Let us finally mention only a few, more recent papers: Aksoy and Maligranda (see [1]) studied descriptions of spaces of Lipschitz-Orlicz type $\operatorname{Lip}\left(\alpha, L_{M}\right)$ and 
$\operatorname{Zyg}\left(\alpha, L_{M}\right)$ in terms of Poisson integrals; Brandolini [5] introduced generalised Lipschitz spaces, i.e. spaces of the type $\Lambda_{X}^{\alpha}\left(\mathbb{R}^{n}\right), \alpha>0$ and $X$ being either $L_{p, \infty}\left(\mathbb{R}^{n}\right)$ or $L_{p}\left(\mathbb{R}^{n}\right)$, given by

$$
\left\|f\left|\Lambda_{X}^{\alpha}\left(\mathbb{R}^{n}\right)\|=\| f\right| X\right\|+\sup _{\delta>0} \frac{\omega_{k}(f, \delta)_{X}}{t^{\alpha}} .
$$

In other words, for $X=L_{p}\left(\mathbb{R}^{n}\right)$ and $\alpha=1$ these are the above spaces $\operatorname{Lip}_{p, \infty}^{(1,0)}$; the modification for $X=L_{p, \infty}\left(\mathbb{R}^{n}\right)$ and arbitrary $\alpha>0$ are clear.

Finally, the closest approach we found in the literature so far - really dealing with logarithmic or similar modifications of the usual Lipschitz spaces - is given in the paper [4] by Bloom and De Souza. They concentrated on weighted Lipschitz spaces of the type Lip $\varrho$, where $\varrho:[0,2 \pi] \rightarrow[0, \infty)$ is a non-decreasing weight function with $\varrho(0)=0$. With a slight modification we may regard $\varrho_{\alpha}(t) \sim t|\log t|^{\alpha} \quad(t>0$ small $)$ as such a weight, belonging to their classes $b_{p}$ for $p>1$, but $\varrho_{\alpha} \notin b_{1}$ (see [4: Definition 1]). Here some weight $\varrho$ is in the class $b_{p} \quad(p \geq 1)$ if

$$
\int_{h}^{2 \pi} \frac{\varrho(t)}{t^{p+1}} d t \leq C \frac{\varrho(h)}{h^{p}} .
$$

Moreover, $\varrho_{\alpha}$ is Dini, as $\int_{0}^{h} \frac{\varrho(t)}{t} d t \leq c \varrho(h)$ for each $h>0$. In their notation we obtain that $\operatorname{Lip} \varrho_{\alpha}=\operatorname{Lip}_{\infty, \infty}^{(1,-\alpha)}$ and for the Zygmund spaces $\Lambda\left(\varrho_{\alpha}\right)=\mathcal{C}^{(1,-\alpha)}$. Recall that similar spaces of the type $C^{0, \sigma(t)}(\bar{\Omega})\left(\Omega \subseteq \mathbb{R}^{n}\right)$ were introduced by Kufner, John and Fučík in [15: Definition 7.2.12, p. 361] (see Remark 3).

\section{References}

[1] Aksoy, A. G. and L. Maligranda: Lipschitz-Orlicz spaces and the Laplace equation. Math. Nachr. 178 (1996), 81 - 101.

[2] Bennett, C. and K. Rudnick: On Lorentz-Zygmund Spaces. Diss. Math. 175 (1980), 1 72 .

[3] Bennett, C. and R. Sharpley: Interpolation of Operators. Boston: Acad. Press 1988.

[4] Bloom, S. and G. S. De Souza: Weighted Lipschitz spaces and their analytic characterizations. Constr. Approx. 10 (1994), 339 - 376.

[5] Brandolini, L.: Imbedding theorems for Lipschitz spaces generated by the weak-L ${ }^{p}$ metric. J. Approx. Theory 94 (1998), 173 - 190.

[6] Brézis, H. and S. Wainger: A note on limiting cases of Sobolev embeddings and convolution inequalities. Comm. Part. Diff. Equ. 5 (1980), 773 - 789.

[7] DeVore, R. and G. G. Lorentz: Constructive Approximation. Berlin: Springer 1993.

[8] Edmunds, D. E. and D. D. Haroske: Embeddings in spaces of Lipschitz type, entropy and approximation numbers, and applications. Report. Jena: Friedrich Schiller Univ., Forschungsergebnisse Math/Inf/98/31 (1998), 1 - 51.

[9] Edmunds, D. E. and D.D. Haroske: Spaces of Lipschitz type, embeddings and entropy numbers. Diss. Math. 380 (1999), $1-43$.

[10] Edmunds, D. E. and H. Triebel: Function Spaces, Entropy Numbers, Differential Operators. Cambridge: Univ. Press 1996. 
[11] Evans, W. D., Opic, B. and L. Pick: Interpolation of operators on scales of generalized Lorentz-Zygmund spaces. Math. Nachr. 182 (1996), $127-181$.

[12] Haroske, D.: Some logarithmic function spaces, entropy numbers, applications to spectral theory. Diss. Math. 373 (1998), 1 - 59.

[13] Haroske, D. D.: Logarithmic Sobolev spaces on $\mathbb{R}^{n}$; entropy numbers, and some application. Forum Math. (to appear).

[14] Krbec, M. and H.-J. Schmeißer: Imbeddings of Brézis-Wainger type. The case of missing derivatives. Preprint. Prague: Math. Inst. Czech Acad. Sci., Preprint 130 (1998), 1 43.

[15] Kufner, A., John, O. and S. Fučík: Function Spaces. Prague: Academia 1977.

[16] Leopold, H.-G.: Limiting embeddings and entropy numbers. Report. Jena: Friedrich Schiller Univ., Forschungsergebnisse Math/Inf/98/05 (1998), 1 - 15.

[17] Lions, P.-L.: Mathematical Topics in Fluid Mechanics. Vol. 2: Compressible Models (Oxford Lect. Ser. Math. \& Appl.: Vol. 10). Oxford: Clarendon Press 1998.

[18] Triebel, H.: Theory of Function Spaces. Basel: Birkhäuser 1983.

[19] Triebel, H.: Theory of Function Spaces II. Basel: Birkhäuser 1992.

[20] Vishik, M.: Hydrodynamics in Besov spaces. Arch. Rat. Mech. Anal. 145 (1998), 197 214.

Received 17.09.1999 